\title{
Mortality of Patients Requiring Escalation to Intensive Care within 24 Hours of Admission in a Mixed Medical-Surgical Population
}

\author{
Jason Leong, MD; Jai Madhok, MD, MSE; and Geoffrey K. Lighthall, MD, PhD
}

\begin{abstract}
Objective: Delayed intensive care unit (ICU) admissions are associated with increased mortality. We present a retrospective study looking at whether indirect admissions to the ICU within 24 hours of hospital admission were associated with increased mortality.

Design: Retrospective cohort study

Setting: Mixed medical-surgical ICU at a large tertiary United States Veterans Affairs (VA) Hospital System
\end{abstract}

Population: The patients were a mix of medical and surgical patients. Patients included both those directly admitted from the operating room as well as those escalated to the ICU after initial admission to the ward (indirect admission).

Methods: All admissions to a medical-surgical ICU from 2008 to 2013 were included in the study. The database was queried for time and location where the admission originated. Separate lists were created for patients with severe sepsis, patients who transferred to the ICU within the first 24 hours, and patients who had rapid response or code team activations. Analysis was applied to the whole group and to medical and surgical subpopulations.

Results: A total of 3,862 ICU admissions were studied. Univariate analysis indicated an impact of delayed admission on whole group and surgical patients; however, multivariate analysis indicated a significant effect of delayed admission on I-year surgical mortality. Multivariate analysis also showed a consistent effect of age, ICU length of stay, and cardiac arrest on mortality of both medical and surgical ICU patients.

Conclusion: In a large retrospective study, surgical patients had increased I-year mortality if they required escalation to the ICU within 24 hours of hospital admission. This result was not replicated in medical patients, possibly related to a burden of illness that could not be altered by earlier care.

Keywords: Deterioration; Intensive care; Mortality; Triage

$\mathrm{I}$ ntensive care units (ICUs) receive critically ill patients at all hours, both directly admitted from the emergency department (ED) or operating room (OR), and also on an unexpected basis from medical-surgical wards following a critical event or deterioration. An early impression of a patient's illness severity and expected course influences whether the patient is admitted to an ICU or medical-surgical ward. These triage decisions are important, as ICU care is a limited resource within any healthcare facility and should be used judiciously. ${ }^{1}$ Studies have shown that patients who have a delay in physical transfer to the ICU due to bed availability have poorer outcomes. ${ }^{2-4}$ It is possible that early triage to a non-ICU unit and eventual transfer may be harmful.

A recent study that looked at ICU utilization in a postoperative surgical population and found indirect admissions to the ICU, defined as patients first being admitted to the floor then transferred to the ICU, were associated with higher
Corresponding Author: Jason Leong, MD, Resident Physician, Department of Internal Medicine \& Anesthesiology, Perioperative and Pain Medicine, 300 Pasteur Dr. H3580, Stanford University School of Medicine, Stanford, CA 94305, Email: jhleong@ stanford.edu
Received: March 12, 2019

Revised: September 3, 2019

Accepted: October 25, 2019

doi: $10.3121 / \mathrm{cmr} .2019 .1497$ 
mortality and morbidity. ${ }^{5}$ Similar findings were noted in a 2014 study of medical patients, where patients who were indirectly admitted to the ICU had higher in-hospital mortality and death within 60 days. ${ }^{6}$ Another study showed that patients with unplanned ICU transfers within 48 hours of admission from an ED who received critical interventions had increased morbidity and mortality. ${ }^{7}$

Different factors associated with ICU admissions have also been associated with higher mortality rates. These characteristics can be split between organizational factors such as time and day of admission, time "delays" in admission to the ICU, ${ }^{8}$ staffing models such as "closed" vs "open" ICUs, ${ }^{9}$ as well as different levels of nursing staffing, ${ }^{10}$ and patientspecific factors such as a age, being a medical or surgical patient (due to differing co-morbidities and health status), being admitted to the ICU for severe sepsis/shock, admission immediately following a respiratory or cardiac arrest, or after a rapid response activation for an acute decompensation. These factors have previously been evaluated for their effects on triage of admissions between intensive care units and other units in the hospital with the goal of identifying patients at risk for poor outcomes.

We report findings of our own retrospective cohort study of a mixed medical-surgical ICU in which we studied the association between indirect ICU admissions within 24 hours of hospital admissions and mortality. This short timeframe ( $<24$ hours) was selected based on prior studies on delays, and also because this is likely to represent deterioration from the initial presenting illness or an incorrect perception of the illness severity and course. This also potentially includes a high-risk patient population that could likely have been triaged to the ICU upon initial presentation.

\section{Methods}

This study was a retrospective analysis of admissions to a mixed medical-surgical ICU at a tertiary referral 325-bed United States Veterans Affairs (VA) hospital for the 64 months between January 1, 2008 and April 30, 2013. This period was chosen because of the availability and fidelity of data for ICU admissions, delayed admission, mortality, and sepsis for these 5 years. The VA Research Committee and the Stanford University Human Subjects panel approved the study.

\section{Patient Population}

All patients admitted from the ward to the ICU were analyzed. The patients were a mix of medical and surgical patients. Patients included both those directly admitted from the operating room as well as those escalated to the ICU after initial admission to the ward (indirect admission).

\section{Data}

The Veterans Affairs Veterans Health Information Systems and Technology Architecture (VISTA) system was queried to generate separate lists of all patients admitted to the ICU, patients that met criteria for severe sepsis at any time during their hospitalization, mortality, all hospital admissions to a non-ICU location, and a list of all patients who passed away in the study timeframe. Additional VA databases were crossreferenced to obtain birthdate and gender information. Code and rapid response team activations were logged into a local database. Patients who had any of the following were classified as having sepsis during that admission: ICD-9 codes for severe sepsis (995.92), septic shock (785.52), or the presence of an infection-related ICD-9 code plus a code for an organ failure or hypotension.

Dates of events such as rapid response activations were compared with ICU admission data to establish relevance to that admission. Rapid response activations within 2 days of ICU admission were considered relevant as a surrogate for detected earlier clinical deterioration. Code events defined as a cardiac or respiratory arrest within 24 hours of ICU admission or during the ICU stay were recorded. Severe sepsis was considered present if the ICD-9 codes mentioned above were generated for the overall hospital admission. Patients admitted to a non-ICU location and subsequently transferred to the ICU within 24 hours of their admission were coded as a "24-hr indirect admissions". Weekend admissions (admission on Saturday or Sunday) were identified for analysis based on prior work demonstrating an increased mortality with this finding. ${ }^{11,12}$ Night admissions were classified as those occurring between 10 PM and 7 AM. Death rates were analyzed as occurring within 30 days, 90 days, or 1 year following ICU admission.

Cases of multiple admissions during the study timeframe were handled in the following manner. If an ICU admission occurred more than 365 days following a prior ICU discharge, the admissions were considered as distinct entities. This convention was followed so patients surviving a year or more following ICU discharge were appropriately classified as such. When multiple ICU admissions occurred within a year of death or discharge date, only the most recent was considered in analysis of survival; these patients were classified as readmissions for purposes of analysis. Thus, the practice of counting only the most recent admission within a given year may inadvertently result in dropping some "24-hr indirect admissions" from analysis, if such an event did not occur in the most recent of the multiple admissions.

\section{Analysis}

Continuous data were analyzed by Analysis Of Variance (ANOVA) or with non-parametric tests for non-normally distributed data. Categorical data were organized into contingency tables and analyzed by Fisher's exact test using Graph Pad Prism (v8.2) software. Stepwise multiple logistic regression was carried out to assess the impact of potentially important clinical variables on 30,90, and 365-day mortality. Variables that showed significant association with bivariate analysis at $P<0.25$ were combined and eliminated in a stepwise manner according to significance level. A correlation matrix was prepared to assess co-linearity of variables; any 
with a correlation coefficient $>0.8$ would be eliminated and described. Some clinical variables deemed to have clinical relevance, even if non-significant, were included in the final analysis. For multivariable analysis, discrete intervals for age were created as follows: 18-40, 41-55, 56-75, 76-85, >85; intervals were based on progressive increases in crude 30-day and 1-year mortality for these groupings. Similarly ICU length of stay was divided into five intervals accordingly as follows: $0-3.2,3.3-7,7-10.5,10.5-14$, and $>14$ days; the reference category was based on the upper confidence interval of the median length of stay (LOS) for all patients studied. Separate analyses were conducted for medical and surgical patients given the mixed medical-surgical nature of our ICU and the generally better survival of surgical ICU patients. Analyses were performed using STATA version14.0 (Stata Corp).

\section{Results}

Medical versus Surgical Patients

There were 3,862 ICU patient encounters analyzed, of which 1,301 were medical and 2,561 were surgical admissions. Demographics and clinical characteristics are shown in Table 1. Overall, medical (vs. surgical) ICU patients had higher illness severity indicators at baseline with higher rates of 30-day mortality ( $25 \%$ vs. $3 \%)$, 90 -day mortality ( $31 \%$ vs. $5.5 \%)$, and 1-year mortality ( $41 \%$ vs. $11 \%$; $P<0.001$ for all). Medical patients also had a higher frequency of 24-hr indirect admissions ( $18 \%$ vs. $7.1 \%$, OR $2.6, P<0.001$ ), readmissions ( $23 \%$ vs. $16 . \%$, OR $1.4, P<0.001)$, code blue events ( $4.2 \%$ vs. $0.4 \%$, OR 10.5 , $P<0.001)$, rapid response calls $(16.3 \%$ vs. $2.7 \%$, OR, 6.0, $P<0.001)$, and presence of severe sepsis ( $27 \%$ vs. $19 \%$, OR 1.4 , $P<0.001)$. Given that surgical patients tend to have more planned ICU admissions during regular work hours, there was more variability in times of admission for medical patients. The differing rates in off-hour admissions were seen in both evening $(25 \%$ vs $12 \%, P<0.001)$ and weekend admissions $(17 \%$ vs $8.8 \%, P<0.0001)$.

The association between a number of clinical conditions and 30and 90-day mortality are shown in Table 2. Except for septic shock, all variables were associated with increased mortality compared to patients without the condition. When medical and surgical patients were considered separately, only rapid response calls $(46 \%$ vs. $38 \%, P=0.021)$ and code blue events $(69 \%$ vs $38 \%, P<0.0001$ ) were associated with a higher 30 -day mortality in medical patients. In contrast, an increased 30-day mortality was associated with both medical and surgical patients with evening admissions $(6.9 \%$ vs. $2.6 \%, P<0.01)$ and ICU readmissions $(7.3 \%$ vs. $2.3 \%, P<0.01)$, as well as code blue events $(55 \%$ vs. $3.2 \%, P<0.01)$ and rapid response calls $(12 \%$ vs. $2.8 \%, P<0.01$; Table $2 \mathrm{~A}$ ). This general difference between medical and surgical patients was maintained in analysis of 90-day mortality (Table 2B) and in 1-year mortality, except for the emergence of an apparent decrease in mortality associated with 24-hour indirect admissions in medical patients for both of these time periods. For mortality at 90 -days, medical patients with indirect ICU admissions had a $24 \%$ mortality vs. $33 \%$ in patients with regular admissions (OR $0.63, P=0.003)$; 1-year mortality was $33 \%$ vs. $41 \%$, OR $0.65 ; P=0.003)$.

\section{Indirect Admissions}

A total of 420 patients, both medical $(n=239)$ and surgical $(\mathrm{n}=181)$, were admitted to the ICU from other hospital locations

Table 1. Demographics and study clinical variables of ICU patients reviewed in the study

\begin{tabular}{lcccl}
\hline $\begin{array}{l}\text { Patient } \\
\text { Characteristic }\end{array}$ & $\begin{array}{c}\text { All patients } \\
(\mathbf{N}=\mathbf{3 8 6 2})\end{array}$ & $\begin{array}{c}\text { Medical patients } \\
\mathbf{( n = 1 3 0 1 )}\end{array}$ & $\begin{array}{c}\text { Surgical patients } \\
(\mathbf{n = 2 5 6 1 )}\end{array}$ & $\begin{array}{l}\boldsymbol{P} \text { value } \\
\text { (Med vs Surg.) }\end{array}$ \\
\hline Age (years \pm SD) & $66.6(12.1)$ & $67.2(13.6)$ & $66.3(11.2)$ & $<0.005^{\star}$ \\
Gender (\% male) & 96.8 & 95.8 & 97.4 & $0.010^{\S}$ \\
ICU LOS (Median, Cl) & $3(2.9-3.2)$ & $3(2.9-3.3)$ & $2.7(2.4-2.9)$ & $<0.0001^{\star \star}$ \\
30d Mortality, $\mathrm{n}(\%)$ & $403(10)$ & $324(25)$ & $79(3.1)$ & $<0.0001^{\S}$ \\
90d Mortality, $\mathrm{n}(\%)$ & $542(14)$ & $401(31)$ & $141(5.5)$ & $<0.0001^{\S}$ \\
1-year mortality, $\mathrm{n}(\%)$ & $809(21)$ & $527(41)$ & $282(11)$ & $<0.0001^{\S}$ \\
24-hr indirect & $420(11)$ & $239(18)$ & $181(7.1)$ & $<0.0001^{\S}$ \\
admission & $65(1.7)$ & $54(4.2)$ & $11(0.4)$ & $<0.0001^{\S}$ \\
Code blue events & $280(7.3)$ & $212(16)$ & $68(2.7)$ & $<0.0001^{\S}$ \\
Rapid response call & $440(11)$ & $215(17)$ & $225(8.8)$ & $<0.0001^{\S}$ \\
Weekend admissions & $630(17)$ & $328(25)$ & $302(12)$ & $<0.0001^{\S}$ \\
Evening admissions & $831(22)$ & $349(27)$ & $482(19)$ & $<0.0001^{\S}$ \\
Severe sepsis & $703(18)$ & $293(23)$ & $411(16)$ & $<0.0001^{\S}$ \\
Readmission & & &
\end{tabular}

Demographics of ICU patients including medical and surgical subsets. *Age was analyzed by Student's t-test; * Length of stay (LOS) was compared by Mann-Whitney test where median and $95 \% \mathrm{Cl}$ for median are shown; §all other categorical variables were analyzed by Fisher's exact test. Abbreviations: ICU, intensive care unit; LOS, length of stay 
where they stayed for less than 24 hours, and were referred here as 24-hr indirect or delayed admissions. The 24-hr indirect admissions had a higher 30 -day mortality of $15 \%$ compared to a $9.9 \%$ mortality in regular admissions (OR 1.39, $P=0.03$ ). The 90 -day mortality difference was similar (20\% vs. $13 \%$, OR 1.46 ; $P=0.005)$. Table 3 looks at the presence of weekend and evening admissions, codes, sepsis, and emergency team calls in patients with direct vs. indirect admissions, and shows that with the exception of sepsis, there is a concentration of these factors in delayed admissions. Rapid response were 7-fold higher in surgical patients with delayed admissions $(P=0.0001)$ and approximately 2.5 -fold higher in medical patients $P<0.0001$; see Table 3). Otherwise, the higher concentration of weekend and evening admissions and code calls were only found in surgical patients Table 3. Of the 420 patients with delayed admissions, 181 (43\%) were surgical and had a significantly higher 90-day and 1 -year mortality than direct admits (11\% vs. 5.1\% at 90-days $P=$ 0.006 , and $18 \%$ vs $11 \%$ at 1 -year, $P=0.003$ ).

\section{Multivariable Analysis}

Given the coincidence of factors influencing mortality, stepwise regression analysis was carried out to analyze clinical variables influencing short and long term mortality in the study population. In addition to the variables considered in the data presented above, age and ICU LOS were entered in as additional variables to control for mortality. Age strata described had a stepwise increase in mortality as shown in the data supplement; LOS was also stratified as described above. Analysis of the whole population found LOS, age, medical admission, cardiac arrest,
ICU readmission, and renal replacement therapy to be independent predictors of 30-day mortality (Table 4A). Delayed admissions did not predict mortality in the whole group analysis. This pattern remained largely unchanged when 90-day and 1-year mortality were examined (supplemental data file, available online); however, the odds ratios associating the variables with mortality decreased. Also the significance of a renal replacement therapy call as an independent predictor of mortality was lost at 1-year.

With medical admissions indicating one of the largest independent risks for mortality in the study (OR 8.4, $P<0.0001$; Table 4A), separate analyses were therefore conducted for medical and surgical subjects. Multivariable analysis of medical patients indicated that only code blue activation, age strata, and ICU LOS predicted 30-day mortality (Table 4B). Analysis of surgical mortality was also predicted by age, arrest, and ICU LOS, but also ICU readmission and evening admission (Table 4C). Analysis did not indicate 24-hour delayed admission to be a significant predictor of 30-day mortality.

Analysis of 90-day and 1-year mortality in medical patients did not indicate any additional significant predictors of mortality (supplemental data file, Table S3C and S3D; available online). In the analysis of surgical patients mortality at 90-days and 1-year, the most consistent predictors of mortality continued to be LOS, age, code, and ICU readmission; nighttime admission became an insignificant factor in the longer term mortality, but 24-hour delayed admission gained significance in the analysis of 1-year mortality (OR1.6s, $P=0.025)$.

Table 2. Mortality among the medical and surgical intensive care unit (ICU) patient population by admission characteristics

\begin{tabular}{|c|c|c|c|c|c|c|}
\hline Variable & $\begin{array}{l}\text { All Patients } \\
\text { n (\%) }\end{array}$ & $P \S$ & $\begin{array}{l}\text { Surgical Patients } \\
\text { n (\%) }\end{array}$ & $P \S$ & $\begin{array}{l}\text { Medical Patients } \\
\text { n (\%) }\end{array}$ & $P \S$ \\
\hline \multicolumn{7}{|l|}{ A. 30-day mortality } \\
\hline 24-hr indirect admission & $63 / 474(13)$ & 0.0366 & 9 (4.9) vs. 70 (2.9) & 0.17 & 54 (24) vs. 270 (27) & 0.41 \\
\hline Code blue event & $41 / 65(63)$ & $<0.0001$ & 6 (55) vs. 73 (3.2) & $<0.01$ & 35 (69) vs. 289 (23) & $<0.01$ \\
\hline Rapid response call & $73 / 280(26)$ & $<0.0001$ & 8 (12) vs. 71 (2.8) & $<0.01$ & 65 (38) vs. 259 (24) & 0.04 \\
\hline Weekend admissions & $64 / 440(15)$ & 0.0037 & 12 (5.3) vs. 67 (2.9) & 0.13 & 52 (32) vs. 272 (25) & 0.86 \\
\hline Evening admissions & $103 / 630(16)$ & $<0.0001$ & 21 (6.9) vs. 58 (2.6) & $<0.01$ & 82 (30) vs. 242 (25) & 1.0 \\
\hline Severe sepsis/ shock & $102 / 831(12)$ & 0.054 & 19 (3.9) vs. 60 (2.9) & 0.24 & 83 (28) vs. 241 (25) & 0.61 \\
\hline ICU Readmission & 109/704 (15) & $<0.0001$ & 30 (7.3) vs. 49 (2.3) & $<0.01$ & 79 (32) vs. 245 (24) & 0.36 \\
\hline \multicolumn{7}{|l|}{ B. 90-day mortality } \\
\hline 24-hr indirect admission & $88 / 474(19)$ & 0.0037 & 19 (11) vs. 122 (5.1) & 0.006 & 69 (24) vs. 232 (33) & 0.003 \\
\hline Code blue event & $43 / 65(66)$ & $<0.0001$ & 6 (55) vs. 135 (5.3) & $<0.0001$ & 37 (69) vs. 364 (29) & $<0.0001$ \\
\hline Rapid response call & $92 / 280(33)$ & $<0.0001$ & 11 (16) vs. 130 (5.2) & 0.001 & $81(38)$ vs. 320 (29) & 0.012 \\
\hline Weekend admissions & $84 / 440(19)$ & 0.0017 & $16(7.1)$ vs. $125(5.4)$ & 0.282 & 68 (31) vs. 333 (31) & 0.012 \\
\hline Evening admissions & $125 / 630(20)$ & $<0.0001$ & 25 (8.3) vs. $116(5.1)$ & 0.03 & $100(30)$ vs. 301 (31) & 0.89 \\
\hline Severe sepsis/ shock & 133/831 (16) & 0.071 & 35 (7.3) vs. 106 (5.1) & 0.075 & 98 (28) vs. 303 (32) & 0.198 \\
\hline ICU Readmission & 142/704 (20) & $<0.0001$ & 47 (11) vs. 94 (4.4) & $<0.0001$ & 95 (32) vs. 306 (30) & 0.51 \\
\hline
\end{tabular}

Association between clinical factors and 30-day (A), and 90-day mortality (B). Variables are listed along with their presence in the population. Numerators indicate the number dying at the specified time (30d, 90d), and denominators indicate the total number identified with the variable (sepsis, for example). Separate analyses for medical and surgical sub-populations are also presented. $\S P$ values indicate significant increases in mortality for patients in each category using Fisher's exact test. Analysis of 1 -year mortality is in the supplemental data file (available online). 
Table 3. Prevalence of key study characteristics in patients with direct and 24-hr indirect intensive care unit (ICU) admissions

\begin{tabular}{cccl}
\hline Admission Characteristic & $\begin{array}{c}\text { Direct admissions } \\
(\mathbf{n = 3 , 4 4 2 )}\end{array}$ & $\begin{array}{c}\text { 24-hr indirect admissions } \\
(\mathbf{n}=\mathbf{4 2 0})\end{array}$ & \multicolumn{1}{c}{$\boldsymbol{P}$ value $^{\S}$} \\
\hline Severe sepsis, $n$ (\%) & $730(21)$ & $101(24)$ & 0.186 \\
Medical & $281(26)$ & $68(28$. & 0.52 \\
Surgical & $449(19)$ & $33(18)$ & 0.92 \\
Evening admissions, $n(\%)$ & $539(16)$ & $91(22)$ & 0.0026 \\
Medical & $271(26)$ & $58(24)$ & 0.74 \\
Surgical & $268(11)$ & $33(18)$ & 0.008 \\
Weekend admissions, $n(\%)$ & $369(11)$ & $71(17)$ & $<0.001$ \\
Medical & $179(17)$ & $36(15)$ & 0.56 \\
Surgical & $190(8.0)$ & $35(19)$ & 0.0001 \\
Code blue, $n$ (\%) & $49(1.4)$ & $16(3.8)$ & $<0.001$ \\
Medical & $41(3.9)$ & $13(5.4)$ & 0.28 \\
Surgical & $8(0.3)$ & $3(1.7)$ & 0.037 \\
Rapid response call, $n(\%)$ & $183(5.3)$ & $97(23)$ & $<0.0001$ \\
Medical & $139(13)$ & $73(31)$ & $<0.0001$ \\
Surgical & $44(1.8)$ & $24(13)$ & 0.0001 \\
\hline
\end{tabular}

Presence of admission characteristics in patients with direct and indirect ICU admissions. The first line of each category indicated the frequency in the whole population, with medical and surgical patients considered separately below. ${ }^{\S} P$ values indicate analysis of categorical variables using Fisher's exact test.

\section{Discussion}

While previous studies have reported higher mortality with delayed ICU admission and indirect ICU admissions, ${ }^{3,5,6,8}$ we conducted this study to evaluate potential differences of this effect between medical and surgical patients and to evaluate the impact of delayed admission on both short- and long-term mortality.

In a multivariate analysis, our study showed a difference only in 1 -year mortality in surgical patients attributable to indirect 24-hour admissions to the ICU. Interestingly, this was not true of the medical patient population, probably related to their high overall 1-year mortality rate compared to surgical patients $(41 \%$ vs. $11 \%$; Table 1). There are many possible reasons for this finding. First, given the higher mortality rate of medical patients in our study, we could have been inadequately powered to see a difference. Second, we are assuming that critical care interventions are initiated solely in the ICU. While this is largely true for multiple vasopressors and invasive ventilation, it is likely that early appropriate interventions such as fluid administration, noninvasive ventilation, and early titration of vasopressors did take place prior to the transfer of medical patients to the ICU, as a large number of indirect medical admissions originated from a step down unit where these capabilities exist and are commonly used. Lastly there may be some inherent difference in patients triaged as stable enough to be admitted to the floor first, as compared to patients who were deemed unstable upon arrival to the ED prompting a direct ICU admission.

A VA study has inherent issues with generalizability given the predominantly male population with similar co-morbidities. ${ }^{13-15}$
However, we believe that this was a strength in our study, as using a relatively homogenous population allowed us to evaluate system-based factors as well as differences between medical and surgical population with fewer confounding factors.

Admissions on weekends for medical and surgical patients trended toward but did not show a significant association with 1-year mortality in our study. A prior large meta-analysis looking at over 97 studies (over 51 million patients) found an association between being admitted on the weekend versus weekday and higher mortality. ${ }^{12}$ This study concluded that weekend admissions were an independent predictor of higher mortality even when taking into account staffing levels and reduced frequency of procedures done on the weekend. Our study could have been inadequately powered to see this association.

We found that surgical patients had higher 1-year mortality for patients who had one of many characteristics including transfer of care to the ICU within 24 hours of admission, being admitted during the night, having a rapid response activation, or a cardiac arrest in comparison to medical patients who only had a higher 1-year mortality for code and rapid response. Overall, the incidence of these characteristics was much higher for medical patients, suggesting a population with a higher severity of illness. Surgical patients had much lower incidence of these characteristics, but when present did lead to higher 1-year mortality. The differences noted again likely speak to differences between medical and surgical patients, wherein the latter are generally healthier, and changes in condition may respond better to early aggressive interventions. This may account for why indirect and nighttime admissions had an impact on mortality. 
Table 4. Multivariable analysis of 30-day mortality in ICU patients

\begin{tabular}{|c|c|c|c|c|}
\hline Variable & OR & SE & 95\% Conf. Int. & $P>[z]$ \\
\hline \multicolumn{5}{|l|}{ A. All patients } \\
\hline LOS Strata & 1.41 & 0.06 & $1.288-1.535$ & 0.0000 \\
\hline Age Strata & 1.87 & 0.13 & $1.622-2.146$ & 0.0000 \\
\hline ICU Readmission & 1.62 & 0.22 & $1.242-2.122$ & 0.0000 \\
\hline Medical vs. Surgical & 8.42 & 1.16 & $6.435-11.02$ & 0.0000 \\
\hline RRT & 1.42 & 0.24 & $1.018-1.991$ & 0.0390 \\
\hline Code Blue & 8.48 & 2.51 & $4.748-15.134$ & 0.0000 \\
\hline 24-hr Delay Admit & 0.84 & 0.14 & $0.599-1.169$ & 0.2960 \\
\hline \multicolumn{5}{|l|}{ B. Medical patients } \\
\hline LOS Strata & 1.34 & 0.07 & $1.209-1.48$ & 0.0000 \\
\hline Code Blue & 6.09 & 1.85 & $3.349-11.055$ & 0.0000 \\
\hline RRT & 1.30 & 0.23 & $0.917-1.853$ & 0.1390 \\
\hline 24-hr Delay Admit & 0.77 & 0.14 & $0.536-1.099$ & 0.1480 \\
\hline Age Strata & 1.68 & 0.13 & $1.438-1.958$ & 0.0000 \\
\hline \multicolumn{5}{|l|}{ C. Surgical patients } \\
\hline LOS Strata & 1.54 & 0.14 & $1.296-1.835$ & 0.000 \\
\hline Age Strata & 2.45 & 0.37 & $1.829-3.294$ & 0.000 \\
\hline ICU Readmission & 2.57 & 0.67 & $1.541-4.282$ & 0.000 \\
\hline PM Admission & 2.36 & 0.67 & $1.356-4.121$ & 0.002 \\
\hline $\mathrm{RRT}$ & 1.72 & 0.81 & $0.68-4.326$ & 0.253 \\
\hline 24-hr Delay Admit & 0.97 & 0.41 & $0.426-2.225$ & 0.950 \\
\hline Code Blue & 30.33 & 20.33 & $8.154-112.838$ & 0.000 \\
\hline
\end{tabular}

Multi-variable logistic regression of clinical characteristics and their impact on 30-day mortality. Analysis of all patients in the study is presented in A, while medical patients are presented in B, and surgical patients are described in C. Abbreviations: OR, odds ratio; SE, standard error of measurement; ICU, intensive care unit; LOS, length of stay; RRT, renal replacement therapy. Analysis of 90-day and 1 -year mortality is presented in the supplemental data file (available online).

Despite best efforts, the disease at hand and the patient's physiologic reserve likely have the greatest impact on survival of medical patients.

A limitation of the present study is the small set of variables available for mortality adjustment. While several computational methods for risk adjustment of ICU patients, such as APACHE, SOFA, and a VA- developed tool exist, ${ }^{16-19}$ we did not have access to these tools for this study. We do, however, acknowledge that they may have helped immeasurably in accounting for co-morbidities and disease burden in our patients.

While our study is limited by confounders present in most retrospective studies, ${ }^{9,10}$ this study does shed some valuable insights into future areas of investigation. First, it appears that ICU admission delays are a separate phenomenon than indirect ICU admissions within 24 hours of presentation. Second, direct admissions postoperatively to the ICU is a practice that varies from institution-to-institution and country of practice due to the limited ICU resources and no true standardization of which patients warrant direct ICU admission post-operatively. ${ }^{20-22}$ Our study found that our surgical population, which included planned post-operative admissions, had higher 1-year mortality associated with 24-hour indirect admissions. This represents the subset of surgical patients who were initially deemed stable enough to be admitted to the floor postoperatively and then required a transfer to the ICU within the first 24 hours. Thus, one could hypothesize that having a deliberate system in place to determine which patients or procedures are high-risk enough requiring planned post-operative ICU admissions could improve outcomes. This observation has been made before in other countries, ${ }^{5}$ but to our knowledge, our study is the first study looking specifically at mortality trends of patients transferred within the first 24 hours post-operatively, when a patient is likely most vulnerable. It is unclear what drives this increase; whether it is from a "failure to rescue", which has been previously studied in surgical patients, ${ }^{21-23}$ that would have been picked up earlier had they already been admitted to the ICU. Another possible explanation is that surgical patients that end up requiring transfer from the ward to the ICU suffer from devastating complications such as a massive pulmonary embolism, post-operative myocardial infarction, hemorrhagic shock, or other complication accounting for higher mortality. Future studies are needed to determine if there are identifiable risk factors (surgical, patient-specific co-morbidities, emergency nature of surgical intervention, etc.) 
that could help guide triage decisions regarding appropriateness of direct ICU admissions from the operating room.

Many societies, such as the Society of Critical Care Medicine, have created guidelines for admissions and a prioritization framework for who should be admitted to the ICU; however, many of them were created with a limited amount of highquality evidence and have not been validated. While a smaller proportion of ICU admissions have clear ICU indications, such as requiring life support from single or multiple organ failure, many ICU admissions fall into a grey zone. Results from our study can hopefully guide future areas of research with regards to ICU admission, triage, and long-term outcomes to help stratify these admissions to best optimize patient outcomes and critical care resource utilization.

\section{Conclusion}

In a large retrospective study of survival of ICU patients, factors such as age, ICU LOS, and arrests had the greatest independent impacts on survival. Some situational variables such as nighttime admission and indirect admission and re-admission to the ICU impacted the survival of surgical patients. Surgical patients also had increased 1-year mortality if they required escalation to the ICU within 24 hours of hospital admission; this result was not replicated in medical patients, a finding possibly related to unmeasured factors including disease burden.

\section{References}

1. Halpern NA, Pastores SM, Greenstein RJ. Critical care medicine in the United States 1985-2000: an analysis of bed numbers, use, and costs. Crit Care Med. 2004;32(6):1254-1259. doi:10.1097/01.ccm.0000128577.31689.4c

2. Goldhill DR, White SA, Sumner A. Physiological values and procedures in the $24 \mathrm{~h}$ before ICU admission from the ward. Anaesthesia. 1999;54(6):529-534. doi:10.1046/j.1365-2044.1999.00837.x

3. Liu V, Kipnis P, Rizk NW, Escobar GJ. Adverse outcomes associated with delayed intensive care unit transfers in an integrated healthcare system. J Hosp Med. 2012;7(3):224-230. doi: $10.1002 / \mathrm{jhm} .964$

4. Mardini L, Lipes J, Jayaraman D. Adverse outcomes associated with delayed intensive care consultation in medical and surgical inpatients. J Crit Care. 2012;27(6):688-693. doi:10.1016/j. jcrc.2012.04.011

5. Gillies MA, Harrison EM, Pearse RM, et al. Intensive care utilization and outcomes after high-risk surgery in Scotland: a population-based cohort study. Br J Anaesth. 2017;118(1):123131. doi:10.1093/bja/aew396

6. Molina JA, Seow E, Heng BH, Chong WF, Ho B. Outcomes of direct and indirect medical intensive care unit admissions from the emergency department of an acute care hospital: a retrospective cohort study. BMJ Open. 2014;4(11):e005553. doi:10.1136/bmjopen-2014-005553

7. Dahn CM, Manasco AT, Breaud AH, et al. A critical analysis of unplanned ICU transfer within 48 hours from ED admission as a quality measure. Am J Emerg Med. 2016;34(8):1505-1510. doi:10.1016/j.ajem.2016.05.009

8. Cardoso LT, Grion CM, Matsuo T, et al. Impact of delayed admission to intensive care units on mortality of critically ill patients: a cohort study. Crit Care. 2011;15(1):R28. doi:10.1186/ cc9975

9. Ghorra S, Reinert SE, Cioffi W, Buczko G, Simms HH. Analysis of the effect of conversion from open to closed surgical intensive care unit. Ann Surg. 1999;229(2):163-171.

\section{doi:10.1097/00000658-199902000-00001}

10. West E, Barron DN, Harrison D, Rafferty AM, Rowan K, Sanderson C. Nurse staffing, medical staffing and mortality in Intensive Care: An observational study. Int J Nurs Stud. 2014;51(5):781-794. doi:10.1016/j.ijnurstu.2014.02.007

11. Pauls LA, Johnson-Paben R, McGready J, Murphy JD, Pronovost PJ, Wu CL. The Weekend Effect in Hospitalized Patients: A Meta-Analysis. J Hosp Med. 2017;12(9):760-766. doi:10.12788/jhm.2815

12. Zajic P, Bauer P, Rhodes A, et al. Weekends affect mortality risk and chance of discharge in critically ill patients: a retrospective study in the Austrian registry for intensive care. Crit Care. 2017;21(1):223. doi:10.1186/s13054-017-1812-0

13. Dominitz JA, Boyko EJ, Koepsell TD, et al. Elevated prevalence of hepatitis C infection in users of United States veterans medical centers. Hepatology. 2005;41(1):88-96. doi:10.1002/ hep. 20502

14. Klevens RM, Giovino GA, Peddicord JP, Nelson DE, Mowery P, Grummer-Strawn L. The association between veteran status and cigarette-smoking behaviors. Am J Prev Med. 1995;11(4):245250.

15. Mapel DW, Dedrick D, Davis K. Trends and cardiovascular co-morbidities of COPD patients in the Veterans Administration Medical System, 1991-1999. COPD. 2005;2(1):35-41. doi:10.1081/copd-200050671

16. de Grooth HJ, Geenen IL, Girbes AR, Vincent JL, Parienti JJ, Oudemans-van Straaten HM. SOFA and mortality endpoints in randomized controlled trials: a systematic review and metaregression analysis. Crit Care. 2017;21(1):38. doi:10.1186/ s13054-017-1609-1

17. Knaus WA, Draper EA, Wagner DP, Zimmerman JE. APACHE II: a severity of disease classification system. Crit Care Med. 1985;13(10):818-829.

18. Render ML, Deddens J, Freyberg R, et al. Veterans Affairs intensive care unit risk adjustment model: validation, updating, recalibration. Crit Care Med. 2008;36(4):10311042. doi:10.1097/ CCM.0b013e318169f290

19. Render ML, Kim HM, Deddens J, et al. Variation in outcomes in Veterans Affairs intensive care units with a computerized severity measure. Crit Care Med. 2005;33(5):930-939. doi:10.1097/01.ccm.0000162497.86229.e9

20. Bui JQ, Mendis RL, van Gelder JM, Sheridan MM, Wright KM, Jaeger M. Is postoperative intensive care unit admission a prerequisite for elective craniotomy?. J Neurosurg. 2011;115(6):1236-1241. doi:10.3171/2011.8.JNS11105

21. Pearse RM, Harrison DA, James P, et al. Identification and characterisation of the high-risk surgical population in the United Kingdom. Crit Care. 2006;10(3):R81. doi:10.1186/cc4928

22. Rhodes A, Moreno RP, Metnitz B, Hochrieser H, Bauer P, Metnitz P. Epidemiology and outcome following post-surgical admission to critical care. Intensive Care Med. 2011;37(9):14661472. doi:10.1007/s00134-011-2299-9

23. Ghaferi AA, Birkmeyer JD, Dimick JB. Variation in hospital mortality associated with inpatient surgery. N Engl J Med. 2009;361(14):1368-1375. doi:10.1056/NEJMsa0903048

\section{Author Affiliations}

Jason Leong, MD*; Jai Madhok, MD, MSE*; Geoffrey K. Lighthall $M D, P h{ }^{\dagger}$

*Resident Physician; Department of Internal Medicine \& Anesthesiology, Perioperative and Pain Medicine, 300 Pasteur Dr. H3580, Stanford University School of Medicine, Stanford, CA 94305 †Professor, Anesthesia and Critical Care; Department of Anesthesia, 300 Pasteur Dr. H3580, Stanford University School of Medicine, Stanford, CA 94305. 